\title{
Viable interspecies chimeras created by injection of Apodemus embryonic stem cells into Mus blastocysts
}

Andy Peng Xiang ${ }^{1, *}$, Frank Fuxiang Mao ${ }^{1,2,{ }^{*}, \text { Donghyun Park }}{ }^{2}$, Bao-Feng Ma ${ }^{1}$, Wei-Qiang Li ${ }^{1}$, Tao Wang ${ }^{1}$, Tammy W Vallender ${ }^{2}$, Eric J Vallender ${ }^{2}$, Li Zhang ${ }^{2}$, Jaehyun Lee ${ }^{2}$, John A Waters ${ }^{3}$, Xiu-Ming Zhang ${ }^{1}$, Xin-Bing $\mathrm{Yu}^{1}$, Shu-Nong $\mathrm{Li}^{1}$, Bruce T Lahn ${ }^{1,2}$

${ }^{1}$ Center for Stem Cell Biology and Tissue Engineering, Sun Yat-sen University, Guangzhou 510080, China; ${ }^{2}$ Howard Hughes Medical Institute, Department of Human Genetics, University of Chicago, Chicago, IL 60637, USA; ${ }^{3}$ Department of Veterinary Clinical Sciences, University of Liverpool, UK

Embryonic stem (ES) cells can differentiate into a variety of specialized cell types when introduced into early embryos (i.e., morulas or blastocysts) of the same species. A question of medical and basic biological importance is whether ES cells of one species can differentiate properly and contribute significantly to chimerism when placed within early embryos of another distantly related species. Here, we address this question using two divergent mammalian model organisms, Apodemus sylvaticus and Mus musculus, whose genomes differ by about $15 \%$. Despite this considerable evolutionary distance, injection of Apodemus ES cells into Mus blastocysts led to viable chimeras bearing extensive Apodemus contributions in all the major organs. Immunostaining showed that Apodemus ES cells had differentiated into a wide range of cell types in the chimeras. These results support the feasibility of deriving a variety of specialized cells or perhaps even complex tissues from ES cells of one species by placing them in the blastocysts of another divergent species. Our data also highlight the remarkable evolutionary conservation of developmental signaling by revealing its compatibility between two rathe distantly related organisms.

Keywords: interspecies, chimera, Apodemus sylvaticus, Mus musculus, embryonic stem cells, blastocyst, microinjection Cell Research (2008) 18:s32. doi: 10.1038/cr.2008.122; published online 4 August 2008

*These two authors contributed equally to this work. Correspondence: Andy Peng Xiang ${ }^{\mathrm{a}}$, Bruce T Lahn ${ }^{\mathrm{b}}$

aE-mail: xiangp@mail.sysu.edu.cn

${ }^{\mathrm{b}}$ E-mail: blahn@bsd.uchicago.edu

Andy Peng Xiang, $\mathrm{PhD}$, Dr Xiang received a PhD in biochemistry and molecular biology from West China University of Medical Sciences and took postdoctoral training in Department of Human Genetics, University of Chicago. He is current Associate Professor in Sun Yat-sen University,
Director of Center for Stem Cell Biology and Tissue Engineering, SYSU. $\mathrm{He}$ has received funding from National Natural Science Foundation of China, the Key Scientific and Technological Projects of Guangdong Province, etc. He has published more than 30 papers in peer-reviewed journals, such as Human Molecular Genetics, Rejuvenation Research. Dr Xiang research focused on the molecular mechanisms of stem cells selfrenewal and multi- differentiation. Please visit his website for more details. http:// www.stemcells.cn 